\title{
Welche Bedeutung hat eine alternde Bevölkerung für das österreichische Steueraufkommen? ${ }^{1}$
}

\author{
Ralf Kronberger ${ }^{*}$
}

\begin{abstract}
Der langfristige Verlauf des Steueraufkommens wird an Hand der demografischen Entwicklung bis 2050 geschätzt. Produktivitätsveränderungen, Verschiebungen in der Erwerbsquote, Veränderungen in der Nettoersatzrate, im Konsum- und Sparverhalten sind auf Grund der demografischen Entwicklung sehr wahrscheinlich. All diese Faktoren dürften einen beträchtlichen Einfluss auf die Entwicklung des Steueraufkommens haben. Mittels der Schätzung unterschiedlicher demografischer Szenarien - vereinzelte beeinflussende Faktoren werden variiert, die Abgabenstruktur bleibt konstant - werden wahrscheinliche Veränderungen im Steueraufkommen analysiert sowie daraus abzuleitende notwendige Politikmaßnahmen diskutiert.
\end{abstract}

\section{Motivation und Gang der Arbeit}

Die wirtschaftspolitische Diskussion wurde in jüngster Zeit und wird nach wie vor stark von sozialpolitischen Themen dominiert. Insbesondere ziehen die Reformen im Gesundheitssektor wie bei den gesetzlichen Pensionen die Aufmerksamkeit der Öffentlichkeit an sich. Auf Grund der nachteiligen demografischen Entwicklung ist mit deutlichen Ausgabensteigerungen vor allem in den zuvor erwähnten Bereichen $\mathrm{zu}$ rechnen. Bei den langfristigen Projektionen zukünftiger Haushaltsabschlüsse werden $\mathrm{zu}$ erwartende Kostensteigerungen meist konstant gehaltenen Einnahmen gegenüber gestellt (z.B. fixer Prozentanteil des BIP): “...the projections of revenues ... would be based on assumptions of unchanged

1 Ich danke Fidelis Bauer, Alexander Hofmann, Reinhard Koman, Robert Koza, Christoph Schneider, Thomas Url, Peter Zacherl für die Bereitstellung von Datenmaterial sowie für wertvolle Kommentare. Ebenso danke ich den zwei anonymen Gutachtern wie auch den Teilnehmern des 1. Workshops aus Finanzwissenschaft der Universität Innsbruck für wertvolle Kommentare zur Arbeit, die dort am 17.1.2003 präsentiert wurde. In diesem Papier geäußerte Meinungen sowie allfällige Fehler und Irrtümer sind ausschließlich jene des Verfassers.

* Wirtschaftskammer Österreich, Wiedner Hauptstraße 63, A-1045 Wien. Email:

ralf.kronberger@wko.at 
shares in GDP over the projection period." (Dang et al. 2001). Implizit wird dabei auf die Berücksichtigung der Angebotsseite verzichtet, die einen wesentlichen Einfluss auf das Steueraufkommen bzw. Beiträge hat. "Also largely ignored in the public debate is the impact of population change on the supply side or productive capacity of the economy, and hence on the ability of the economy to generate tax revenue to support higher expenditure levels." (Denton und Spencer 1999). Es ist Ziel dieser Arbeit zu prüfen, ob die Annahmen der konstant gehaltenen Beitragsbzw. Aufkommensseite beibehalten werden können. Wird beispielsweise das Aufkommen überschätzt, verringert sich der finanzpolitische Spielraum noch mehr als das Gros der Simulationen bereits suggeriert. Mögliche Konsequenzen einer solchen Fehleinschätzung wären, dass der ohnehin große Druck auf die öffentlichen Haushalte durch die beiden Maastricht-Kriterien - das Defizit der öffentlichen Haushalte soll 3\% des BIP und die öffentliche Verschuldung soll 60\% des BIP nicht überschreiten - weiter erhöht würde. Ausgabenseitig hätten noch größere Rücknahmen zu erfolgen, die insbesondere unter konjunkturell schwierigen Situationen zu einer prozyklischen Fiskalpolitik führen können, wenn es verabsäumt wird, das Aufkommen auf einen nachhaltigen Pfad zu bringen, beziehungsweise dort zu halten. Mittelfristig sollen zudem die EU-Mitgliedstaaten dafür sorgen, dass ihre Haushalte ausgeglichen sind.

Die Arbeit ist wie folgt gegliedert: in Abschnitt zwei erfolgt eine Darstellung existierender Arbeiten, die eine längerfristige Betrachtung des Steueraufkommens in Österreich und Deutschland zum Inhalt haben. Ebenso erfolgt eine kurze Darstellung erwarteter makroökonomischer Effekte der demografischen Veränderung. Abschnitt drei ist in eine Beschreibung der verwendeten Methode, der demografischen Szenarien, der angewandten Profile sowie in eine Darstellung der Ergebnisse gegliedert. In Abschnitt vier erfolgt eine Diskussion der Ergebnisse. Weitere Steuern werden betrachtet und mögliche Maßnahmen zur Sicherung des langfristigen Aufkommens aufgezeigt.

\section{Existierende Arbeiten}

Jüngere Arbeiten, die sich mit dem längerfristigen Steueraufkommen in Österreich beschäftigen, sind Lehner (2002), Lehner (2003), Keuschnigg et al. (2000) und Keuschnigg et al. (2002). Für Deutschland haben Bach et al. (2002) das längerfristige Steueraufkommen berechnet.

Lehner (2002) prognostiziert das Steueraufkommen an Hand eines Elastizitätenansatzes bis zum Jahr 2010. Bei den wirtschaftlichen Rahmenbedingungen geht der Autor davon aus, dass das nominelle Lohnwachstum hinter dem nominellen BIP-Wachstum zurückbleibt, das einmal mit 3,5\% und einmal mit $4 \%$ angenommen wurde. Bei den Pensionen ist die Kaufkraft durch volle Inflationsanpassung gesichert. Zudem werden die Aufkommenselastizitäten auf Basis des ESVG 95 berechnet, das beispielsweise bei der Lohnsteuer zum 
Tabelle 1:

Abgabenstruktur 1999-2001 und 3-Jahres-Durchschnitt

\begin{tabular}{|c|c|c|c|c|c|c|}
\hline \multicolumn{2}{|c|}{ Abgabenstruktur } & \multirow{2}{*}{\begin{tabular}{|c|}
1999 \\
23.487
\end{tabular}} & \multirow{2}{*}{\begin{tabular}{|l}
2000 \\
23.852
\end{tabular}} & \multirow{2}{*}{\begin{tabular}{|c|}
2001 \\
28.780
\end{tabular}} & \multicolumn{2}{|c|}{$\begin{array}{l}\text { 3jahresschnitt } \\
-1999 / 2001\end{array}$} \\
\hline \multirow{2}{*}{\multicolumn{2}{|c|}{$\begin{array}{l}\text { Einkommen } \\
\begin{array}{ll}\text { darunter } & \text { und Vermögensteuern insgesamt } \\
\text { veranlagte Einkommenst. }\end{array}\end{array}$}} & & & & 25.373 & $(\%)$ \\
\hline & & 2.896 & 2.818 & 3.987 & 3.233 & $5,8 \%$ \\
\hline & Lohnsteuer & 14.753 & 14.468 & 16.362 & 15.194 & $27,0 \%$ \\
\hline & Körperschaftst & 3.247 & 3.865 & 6.236 & 4.449 & $7,9 \%$ \\
\hline & Kapitaler & 1.833 & 1.945 & 2.048 & 1.942 & $3,5 \%$ \\
\hline \multicolumn{2}{|c|}{ Umsatzsteuer } & 16.493 & 17.056 & 17.354 & 16.968 & $30,2 \%$ \\
\hline \multicolumn{2}{|c|}{ Verbrauchsteuern insgesamt } & 4.106 & 424 & 4.411 & 2.980 & \\
\hline \multirow[t]{2}{*}{ darunter } & Tabaksteuer & 1.157 & 1.095 & 1.234 & 1.162 & $2,1 \%$ \\
\hline & Mineralölsteuer & 2.695 & 2.726 & 2.880 & 2.767 & $4,9 \%$ \\
\hline \multirow{2}{*}{\multicolumn{2}{|c|}{$\begin{array}{l}\text { Einfuhrabgaben, Gebühren und Verkehrssteuern } \\
\text { Öffentliche Abgaben (Brutto) }\end{array}$}} & 4.589 & 5.240 & 5.666 & 5.165 & $9,2 \%$ \\
\hline & & 48.675 & $\mathbf{5 0 . 3 8 7}$ & 56.211 & 51.757 & \\
\hline \multicolumn{2}{|c|}{ Summe Lohnst. \& Ust } & 31.246 & 31.524 & 33.716 & 32.162 & $62,1 \%$ \\
\hline \multicolumn{2}{|c|}{ Summe Lohnst. \& Ust (Berechnungsgrundlage) } & 30.408 & 30.702 & 32.786 & 31.298 & $60,5 \%$ \\
\hline
\end{tabular}

tatsächlichen Aufkommen zusätzlich Kinderabsetzbeträge, Bausparprämien und Mietzinsbeihilfen berücksichtigt. Bei der Lohnsteuer - sie ist mit 27\% der Bruttoabgaben der zweitgrößte Aufkommensposten hinter der Umsatzsteuer (30,2\%; Tabelle 1) - ermittelt Lehner eine Aufkommenselastizität zum BIP von 1,21. Bei der Körperschaftsteuer verhält sich das Aufkommen proportional zum BIP-Wachstum. Bei der Einkommenssteuer wird von einer leicht überproportionalen Steigerung $(1,08)$ ausgegangen. Beim KESt-Aufkommen wird ebenso von einer leicht überproportionalen Elastizität zum BIP ausgegangen $(1,07){ }^{2}$ Die Umsatzsteueraufkommenselastizität wird bis 2010 auf knapp 0,88 geschätzt. Die Abgabenquote gemäß ESVG 95 fällt auf Basis der errechneten Elastizitäten von 45,9\% des BIP im Jahr 2001 auf 43,9\% im Jahr 2010. Eine Darstellung der verwendeten Methoden erfolgt weder in Lehner (2002) noch in Lehner (2003). Einziger Hinweis ist, dass die Elastizitäten auf Basis unveränderter Rechtslage des Jahres 2002 geschätzt werden und für die drei großen betrachteten Steuergruppen (Bundessteuern, Sozialversicherungsbeiträge, ausschließliche Landes- und Gemeindeabgaben inklusive Kammerumlagen) nicht nur der Zusammenhang mit dem BIP auf Basis der mittelfristigen Konjunktur- und Wachstumsprognosen sondern auch mit dem nominellen Konsum, den Pro-KopfLöhnen und der Beschäftigung im Modell berücksichtigt werden. Der Elastizitätenansatz zeigt bereits bei der Prognose bis 2010 erhebliche Unsicherheiten. Der Autor selbst führt an, dass für die Jahre 2005 bis 2010 keine detaillierten Vorausschauen des Wifo vorliegen.

2 Die Lohnsteuer, die Körperschaftsteuer, die veranlagte Einkommensteuer sowie die Kapitalertragsteuer werden den Einkommen- und Vermögenssteuern zugezählt. 
Eine Fortschreibung der Elastizitäten bis 2050 anstatt bis 2010 würde einerseits entsprechend größere Unsicherheiten auf Grund des 9-fachen Prognosehorizonts bringen, ${ }^{3}$ und andererseits ist davon auszugehen, dass das verwendete Modell demografische Veränderungen nicht berücksichtigt: „Für die Jahre 2005/2010 wird je nach Annahme über das reale Wirtschaftswachstum mit einer Steigerung der Beschäftigung zwischen $0,8 \%$ und 1,0\% zu rechnen sein." (Lehner 2002, S. 9).

Keuschnigg et al. (2002) errechnen die implizite Staatsschuld an Hand einer Generationenbilanz. Sie stellen gegenwärtige und zukünftige Staatseinnahmen den respektiven Staatsausgaben gegenüber. Die Autoren nehmen eine "demografische" Zerlegung der Staatshaushalte vor, um deren Sensitivität auf demografische Änderungen zu ermitteln. Die Methode baut auf Raffelhüschen (1999) auf. Auf der Einnahmenseite werden die Aufkommensarten Lohnsteuern, Sozialversicherungsbeiträge, Kapitalertragsteuer und Umsatzsteuer berücksichtigt. Das von den Autoren verwendete Datenmaterial beruht auf der Lohnsteuerstatistik 1995, der Konsumerhebung 1984, dem Haushaltspanel der Europäischen Union 1995 und dem Demographic Yearbook 1996. Ebenso wie bei anderen Studien wird das Wachstum der Einkommens- und Ausgabenprojektionen konstant gehalten. Die Entwicklung des Steueraufkommens wird in der österreichischen Studie nicht gesondert ausgewiesen.

Bach et al. (2002) verwenden für die Berechnung des längerfristigen Steueraufkommens in Deutschland ebenfalls das Modell der Generationenbilanzierung analog zu Raffelhüschen (1999). Die Autoren kommen dabei zu dem Schluss, dass "Alles in allem ... die längerfristige Entwicklung des Steueraufkommens vom demographischen Wandel nicht sehr stark betroffen sein (wird)". Bei der Einkommenssteuer verändert sich das Aufkommen zwischen 2005 und 2050 unter Zugrundelegung von 4 Prognoseszenarien mit sich verändernder Erwerbsquote in einer Bandbreite von $6,6 \%$ und $12,8 \%$. Bei der Umsatzsteuer beträgt diese Bandbreite $6,7 \%$ bis $12,1 \%$. Das Aufkommen insgesamt ändert sich zwischen $9,2 \%$ und $14,8 \%$. Die unternehmensbezogenen Steuern entwickeln sich vergleichsweise am besten mit Veränderungen zwischen 2,2\% und 7,8\%. ${ }^{4}$

Bei der Analyse der Auswirkungen der demografischen Entwicklungen greift ökonomisch gesehen - eine rein statische Betrachtung beispielsweise über hochgerechnete Einkommens-, Konsum- oder anderer Steuerprofile - wie sie bei Keuschnigg et al. (2002) und Bach et al. (2002) angewandt wurde - zu kurz. Ausgewählte Mikro- und Makrovariablen wie Wertschöpfung, Sparen,

Für eine Diskussion der Steueraufkommenselastizitäten siehe u.a. Leibrecht (2002). Eine Fortschreibung der Gesamt-Aufkommenselastizität von 0,93 würde - bei angenommenem durchschnittlichen nominellen Wachstum von 4\% - die Steuer- und Abgabenquote bis zum Jahr 2050 von 44,4\% auf ein Niveau von um 10\% senken. Auf Veränderungen in der Wachstumsrate reagiert bei so einem langen Prognosehorizont die Abgabenquote verhältnismäßig stark.

4 Baumgartner et al. (2004) erzielen ein reales Wachstum der USt von ca. 2\% bis zum Jahr 2050. Die Lohnsteuer verringert sich real zwischen 0,3 und $0,9 \%$. Für eine kurze Beschreibung des Modells siehe folgende Fußnote. 
Kapitalstock, Löhne werden u.a. durch die veränderten Erwerbsquote und verändertes Sparverhalten bedingt durch die demografische Entwicklung beeinflusst. Diese Effekte können an sich am akkuratesten mit Hilfe von makroökonometrischen Modellen geschätzt werden. ${ }^{5}$

Die über andere Staaten vorliegenden Modellrechnungen lassen folgende ökonomische Auswirkungen der Überalterung erwarten:

- das Wirtschaftswachstum verlangsamt sich;

- der Anstieg des Wohlstands i.S. des Pro-Kopf-Einkommens verlangsamt sich;

- privates Sparen geht auf Makroebene zurück, auf Mikroebene existiert widersprüchliche empirische Evidenz.

Die relativ sinkende Erwerbsbevölkerung wird wahrscheinlich nicht durch ausreichend steigende Investitionen ausgeglichen. Das Anwachsen des Kapitalstocks und technologischer Fortschritt werden voraussichtlich nicht genügen, um der stagnierenden Arbeitsproduktivität entgegen zu wirken (Group of Ten 1998). Denton und Spencer (1999) gehen davon aus, dass langsamer wachsende Investitionen und weniger schnell wachsende Kapitalnachfrage sinkende Kapitalerträge äbedingen. Es ist also damit $\mathrm{zu}$ rechnen, dass das Produktivitätswachstum vergangener Perioden nicht für eine alternde Bevölkerung in die Zukunft projiziert werden kann. ${ }^{6}$

Großen Einfluss auf das Sparverhalten wird die Pensionsfinanzierung haben. Bei groß angelegten Umstellungen von Umlage- auf Kapitaldeckungsverfahren würde der aktuell sinkenden privaten Sparquote entsprechend entgegen gesteuert werden. Die Form der Besteuerung der Pensionszahlungen würde dann erheblichen Einfluss auf das Steueraufkommen entweder bei der Kapitalertragsbzw. Lohnsteuer haben. Erhöhtes Sparen wiederum könnte dämpfende Effekte auf den Konsum vor allem der Erwerbsbevölkerung bedingen. Gedämpftes Wachstum und reduziertes Umsatzsteueraufkommen - zumindest in der Übergangsphase könnten die Folge sein. ${ }^{6}$

5 Baumgartner et al. (2004) rechnen ein langfristiges Makromodell für Österreich bis zum Jahr 2075. Die Bedingung für die öffentlichen Haushalte ist, dass sie langfristig ausgeglichen sind. Das USt-Aufkommen entwickelt sich proportional zum BIP. Für die Lohnsteuer kommt ein durchschnittlicher Steuersatz zur Anwendung. In der Simulation wird begründet durch die Pensionsreform mit steigenden Erwerbsquoten gerechnet. Negative Auswirkungen auf das Wachstum sind nur dann wesentlich spürbar, wenn die Fertilitätsrate spürbar sinkt. Die Bedingung eines ausgeglichenen Sozialversicherungshaushalts mit Indizierung der Pensionsversicherungsbeiträge resultiert in einem leicht geringfügigeren Wachstum und in einer deutlich höheren Arbeitslosigkeit. Die stärkste Wachstumsdämpfung resultiert jedoch aus einer niedrigen Fertilitätsrate.

6 Nicht nur eine veränderte Produktivität ist wahrscheinlich, sondern auch Verschiebungen innerhalb der Verwendungsseite der Wertschöpfung (Denton und Spencer, 1999).

$6 \quad$ Vgl. dazu Wagner et al. (1998) 


\section{Modellrechnung}

\subsection{Methode und Annahmen}

Ähnlich zu Bach et al. (2002) wird das Steueraufkommen wie folgt berechnet:

Das Steueraufkommen $A$ im Jahr $t$ setzt sich aus den Steuerzahlungen $S$ der jeweiligen Überlebenden der Kohorte $k$ zusammen, deren Anzahl $K$ beschreibt.

$$
A_{t}=\sum_{k} S_{t, k} K_{t, k}
$$

Die Steuern sind aufgesplittet in $i$ einzelne Steuern $E$ und werden zu $S$ aufsummiert.

$$
S_{t, k}=\sum_{i} E_{t, k, i}
$$

Von der Prognose und Berücksichtigung des Produktivitätswachstums sowie der Inflation ${ }^{7}$ wird vorerst Abstand genommen. Der Autor orientiert sich dabei an Bach et al.(2002), die bei ihrer Aufkommensschätzung für Deutschland eine Aufkommenselastizität von $1 \mathrm{zu}$ Grunde legen. Sie stellen Veränderungen im Aufkommen ähnlich dieser Arbeit großteils wachstumsbereinigt dar.

Obzwar es sich bei dieser Arbeit um eine Prognose der Einnahmenseite handelt, werden auch vereinzelt Annahmen über die Ausgabeseite getroffen. Beispielsweise ist die Ausgabenseite für Pensionen (Höhe und Anteil der Versicherungsbeiträge) von Belang, insofern als sie für Pensionseinkommen und in Folge für den Konsum der Pensionisten eine Rolle spielt. Gemäß Venti und Wise (2001) werden die Pensionen zur Gänze für den laufenden Konsum der Pensionisten aufgewendet. Bei sinkenden Pensionseinkommen impliziert dies niedrigere Einnahmen aus der Pensionsbesteuerung und weniger Mehrwertsteueraufkommen durch reduzierten Konsum. ${ }^{8}$

Diese Annahme steht in Analogie zur Mackenrothschen These (Mackenroth 1952): der Konsum der Pensionisten finanziert sich immer - unabhängig vom Finanzierungsverfahren -aus dem gegenwärtigen Bruttosozialprodukt. Die

Bei progressiven Steuern bewirkt die Einführung von Inflation einen Aufwärtsdruck auf die Aufkommenselastizität, wenn die Steuergrenzen nicht ausreichend an die Inflation angepasst werden. Dadurch ergibt sich tendenziell ein positiver Impuls für das Aufkommen progressiver Steuern auf lange Sicht.

$8 \quad$ Vergleiche dazu in der aktuellen Literatur Gruber und Wise (2001, 17f.): "This finding suggests that, if current trends continue, the brunt of the burden of an aging society will be borne through reduced spending elsewhere, including on the non-elderly.... This finding is good news for fiscal balances, but potentially bad news for the non-elderly." 
Mackenrothsche These hat vor allem im deutschsprachigen Raum zu einer intensiven Debatte geführt. Kritisiert wird an dieser These, dass sie sich am Bruttosozialprodukt statt am Volkseinkommen orientiert. Ein Szenario ist dadurch denkbar, dass durch einen schrittweisen Abbau des Kapitalstocks der Konsum gesteigert wird. Zudem geht Mackenroth von einer geschlossenen Volkswirtschaft aus. Dies widerspricht der Möglichkeit, dass Kapital für die Pensionen im Ausland angespart werden kann. ${ }^{9}$

Im Folgenden wird von der Annahme ausgegangen, dass nur das Einkommen aus der gegenwärtigen Wertschöpfung zwischen den Erwerbstätigen und Pensionisten aufgeteilt werden kann. Eine Erhöhung des Anteils der Pensionisten führt entweder dazu, dass von den Erwerbstätigen größere Einkommen zu den Pensionisten transferiert werden, zu Lasten ihres eigenen Konsums, oder die Pensionisten mit einem geringeren Einkommen und damit weniger Konsum ihr Auslangen finden müssen. Dazu wird die Beschränkung eingeführt, dass der Konsum der Pensionisten deren laufende Einnahmen nicht überschreiten kann.

Die Pensionseinkommen der Pensionisten $P E$ zum Zeitpunkt $t$ setzen sich zusammen aus den Pensionsversicherungsbeiträgen $P V B$ der unterschiedlichen Kohorten $\mathrm{k}$ mit der Anzahl $\mathrm{K}$, dem Bundesbeitrag $B$, der über den Beobachtungszeitraum konstant gehalten wird sowie der Eigenfinanzierung $E F$, die einen stabilen Pro-Kopf-Betrag für die Kohorten $k$ - $n$ bis $k$ (Pensionisten) für die Anzahl $K$ darstellt.

$$
P E_{t}=B+\sum_{k} P V B_{t, k} K_{t, k}+\sum_{k-n}^{k} E F \cdot K_{t, k}
$$

Die Pensionsausgaben $P A$ entsprechen dem Konsum $K O$ der Kohorten $k-n$ bis $k$ mit der Anzahl $K$.

$$
P A_{t}=\sum_{k-n}^{k} K O_{t, k} \cdot K_{t, k}
$$

Die Beschränkung ergibt sich aus der Identität, dass die Pensionsausgaben den Pensionseinkommen entsprechen.

$$
P A_{t}=P E_{t}
$$

Durch die Einführung der Beschränkung der Einnahmenseite der Pensionisten entsteht eine Beschränkung ihrer Konsumausgaben, die in (1) und (2) durch konstante Fortschreibung der USt- und Lohnsteuerprofile nicht gegeben war. ${ }^{10}$ Das

\footnotetext{
Vgl. dazu auch Keller (2000, 78ff.), Külp (1993), Werding (1998), Holzmann (2000).

10 Die Beschränkung für die Einkommensteuer wird vereinfacht proportional zur Konsumbeschränkung angenommen.
} 
Steueraufkommen mit der eingeführten Beschränkung $A_{t}^{B}$ ist in (6) niedergeschrieben.

$$
A_{t}^{B}=\sum_{k} S_{t, k}^{B} K_{t, k}
$$

Die Differenz zwischen den beiden Modellen Steueraufkommen ohne Konsumbeschränkung mit konstanten Profilen (1) und Steueraufkommen mit Konsumbeschränkung (6) ergibt den Risikoanteil.

Bei der vorliegenden Modellrechnung handelt es sich um eine statische Rechnung, die sich über einen großen Prognosehorizont erstreckt. Prognosen über eine so lange Periode sind grundsätzlich sehr unsicher. Ökonomische Entwicklungen und Politikänderungen können schwerlich vorhergesagt werden. Bereits vorliegende Arbeiten lassen den Schluss zu, dass hohe Schwankungsbreiten zu berücksichtigen sind und die Sensitivität der Ergebnisse stark von den Prognosemethoden und den verwendeten Systemparametern abhängt. Einfache Extrapolationen, statische Berechnungen bzw. partielle Gleichgewichtsmodelle unterliegen allgemeinen Gleichgewichtsmodellen (Visco 2001, S. 4f.).

\subsection{Daten}

\subsubsection{Demografische Prognosen}

Im Jahr 2000 stieg die Statistik Austria auf ein neues Prognosemodell bei der Bevölkerungsvorausschätzung um. Gegenüber zeitlich zurückliegenden demografischen Prognosen wird nun anstatt einer Stagnation des Bevölkerungswachstums von 2000 bis 2030 von einem Bevölkerungswachstum von rund 270.000 Personen ausgegangen. Dies ist durch die gegenüber dem Zeitraum 1995 bis 1998 gestiegene Fertilität wie auch durch höhere Zuwanderung begründet. Neben der mittleren Hauptvariante werden insgesamt zwölf Alternativvarianten geschätzt, die in den Annahmen zu Fertilität, Mortalität und Migration variieren.

Für die Berechung des Steueraufkommens werden neben der Hauptvariante vier alternative Prognosevarianten ausgewählt: ${ }^{11}$ Die Hauptvariante (Haupt) oder mittlere Variante stellt das wahrscheinlichste Prognoseszenario dar. Dabei wird von einer Fertilitätsrate von 1,5 in den Jahren 2015 bis 2050 ausgegangen. Sowohl in der Hauptvariante als auch in den nachfolgend beschriebenen Varianten mit niedriger und hoher Fruchtbarkeit wird angenommen, dass die regionalen Fertilitätsniveaus bis zum Jahr 2020 um ein Drittel des Ausgangsniveaus des

11 Für eine umfassendere Darstellung zur demographischen Prognose siehe Hanika (2000). 
Zeitraumes 1995/99 konvergieren. ${ }^{12}$ Die Lebenserwartung liegt 2050 bei den Männern bei 82 Jahren und bei den Frauen bei 87 Jahren. Langfristig reduziert sich in der Hauptvariante die Differenz bei der Lebenserwartung zwischen Männern und Frauen von derzeit knapp sechs auf fünf Jahre.

Die Migration wird nicht in Form eines Nettomigrationssaldos geschätzt, sondern an Hand der internationalen Zuwanderung, der internationalen Abwanderung und der Binnenwanderungsströme. Die Emigration geschieht im Wesentlichen durch Kinder, Jugendliche und jüngere Erwachsene, wodurch durch zunehmende Überalterung zu erwarten ist, dass die Zahl der Emigranten längerfristig tendenziell sinken wird (in der mittleren Variante von $67.000 \mathrm{im} \mathrm{Jahr}$ 1999 auf 57.000 im Jahr 2050). Die Zuwanderung belief sich im Jahr 1999 auf 87.000 Personen. Die Prognose der Zuwanderung ist von verhältnismäßig großer Unsicherheit geprägt, da schwer abschätzbar ist, wie die Asyl- und Migrationspolitik fortgesetzt wird. Weiters ist vermehrte Zuwanderung aus den östlichen Nachbarstaaten durch deren EU-Beitritt und durch das sich verknappende Arbeitskräfteangebot im Inland infolge des Überalterungsprozesses zu rechnen. Die durchschnittliche internationale Zuwanderung wird in der mittleren Variante zwischen 2020 und 2050 mit 80.000 Personen jährlich angenommen. Im Zeitraum 2020 bis 2030 würde diese einer Nettowanderungsannahme von rund 20.000 Personen entsprechen.

In der Benchmarkvariante (Benchmark) erfolgt eine Fortschreibung der demografischen Daten von 1999 ohne Variation der bereits oben angeführten Parameter (Gesamtfertilität 1,1, Lebenserwartung der Männer 75,1 Jahre, Lebenserwartung der Frauen 80,9 Jahre, 87.000 Zuwanderer jährlich).

Die Variante mit hoher Fruchtbarkeit und hoher Wanderung (+Fruchtbarkeit +Wanderung) zeigt ein verhältnismäßig günstiges Szenario hinsichtlich der Bevölkerungsalterung. Die Gesamtfertilität wird mit 1,8 zwischen 2020 und 2050 angenommen, die internationale Zuwanderung im selben Zeitraum mit 100.000 Personen jährlich, also einem Plus von rund 20.000 Personen gegenüber der mittleren Variante.

Die Variante mit niedriger Fruchtbarkeit und niedriger Wanderung (-Fruchtbarkeit-Wanderung) geht von einer Gesamtfertilität von 1,2 und einer internationalen jährlichen Zuwanderung von 60.000 Personen aus, diesmal ein Minus von 20.000 Personen gegenüber der mittleren Variante.

Bei der Variante mit hoher Lebenserwartung (+Lebenserwartung) liegt eine Lebenserwartung der Männer von 86 und der Frauen von 90 Jahren für das Jahr $2050 \mathrm{zu}$ Grunde. Im Unterschied zur Hauptvariante verringert sich die Differenz bei der Lebenserwartung zwischen Männern und Frauen von derzeit knapp sechs auf vier Jahre. Die restlichen Parameter entsprechen der mittleren Variante.

Die Altenbelastungsquote wird im Allgemeinen als Maß geführt, das angibt, in wie weit die erwerbsfähige Bevölkerung für die über 59-Jährigen zu sorgen hat. Für die nachfolgende Berechnung ist sie aus zweierlei Gründen relevant. Erstens

12 Keuschnigg et al. (2000) halten demographische Prognoseparameter bereits ab 2010 konstant. 
ist davon auszugehen, dass die Steuerleistung durch die Zunahme der über 59-Jährigen durch geringeres laufendes Einkommen (Lohnsteuer) und durch geringeren Konsum (Umsatzsteuer) dieser Kohorten insgesamt abnehmen wird. Zweitens ist unter der Annahme, dass der Konsum der über 59-Jährigen aus dem laufenden Einkommen der angesprochenen Kohorte, das überwiegend umlagefinanziert ist, eine große demografische Reagibilität der Einkommen und folglich des Konsums der über 59-Jährigen auf die Altenbelastungsquote gegeben. Entsprechend den Parametern fallen die Altenbelastungsquoten der Relation von über 59-Jährigen zu 15- bis 59-Jährigen aus. Ausgehend von 34\% im Jahr 2001 steigt sie im Hauptszenario bis zum Jahr 2050 auf 66,9\% (Tabelle 2). Am niedrigsten ist sie im Jahr 2050 für die Benchmarkvariante mit 58,0\% knapp gefolgt von der +Fruchtbarkeit+Wanderung-Variante mit 58,4\%. Bei der -Fruchtbarkeit-Wanderung-Variante steigt sie bis zum Jahr 2050 auf 78,4\%.

Tabelle 2:

Altenquote: Anteil der über 59-Jährigen gemessen an den erwerbsfähigen Personen (15- bis 59-Jährige)

\begin{tabular}{|c|c|c|c|c|c|c|}
\hline \multicolumn{7}{|c|}{ Bevölkerungsentwicklung und Altenquote } \\
\hline & 2001 & 2010 & 2020 & 2030 & 2040 & 2050 \\
\hline Haupt & $34,0 \%$ & $38,1 \%$ & $45,6 \%$ & $59,8 \%$ & $64,5 \%$ & $66,9 \%$ \\
\hline -Fruchtbarkeit & & & & & & \\
\hline $\begin{array}{l}\text {-Wanderung } \\
\text { +Fruchtbarkeit }\end{array}$ & $34,0 \%$ & $38,4 \%$ & $46,8 \%$ & $64,6 \%$ & $73,1 \%$ & $78,4 \%$ \\
\hline +Wanderung & $34,0 \%$ & $37,9 \%$ & $44,3 \%$ & $55,6 \%$ & $57,9 \%$ & $58,4 \%$ \\
\hline Benchmark & $34,0 \%$ & $37,5 \%$ & $43,3 \%$ & $55,1 \%$ & $57,5 \%$ & $58,0 \%$ \\
\hline +Lebenserwarung & $34,0 \%$ & $38,3 \%$ & $46,4 \%$ & $61,8 \%$ & $68,3 \%$ & $73,0 \%$ \\
\hline \multicolumn{7}{|c|}{ Anzahl über 59jähriger (in Tausend) } \\
\hline Haupt & 1.723 & 1.942 & 2.237 & 2.701 & 2.840 & 2.856 \\
\hline $\begin{array}{l}\text {-Fruchtbarkeit } \\
\text {-Wanderung } \\
\text { +Fruchtbarkeit }\end{array}$ & 1.723 & 1.939 & 2.221 & 2.657 & 2.750 & 2.696 \\
\hline +Wanderung & 1.723 & 1.945 & 2.254 & 2.745 & 2.929 & 3.015 \\
\hline Benchmark & 1.723 & 1.909 & 2.118 & 2.441 & 2.426 & 2.321 \\
\hline +Lebenserwartung & 1.723 & 1.953 & 2.280 & 2.798 & 3.015 & 3.131 \\
\hline \multicolumn{7}{|c|}{ Bevölkerungsentwicklung gesamt (in Tausend) } \\
\hline Haupt & 8.128 & 8.227 & 8.323 & 8.386 & 8.344 & 8.214 \\
\hline $\begin{array}{l}\text {-Fruchtbarkeit } \\
\text {-Wanderung } \\
\text { +Fruchtbarkeit }\end{array}$ & 8.127 & 8.116 & 7.926 & 7.654 & 7.289 & 6.836 \\
\hline +Wanderung & 8.130 & 8.366 & 8.739 & 9.158 & 9.487 & 9.734 \\
\hline Benchmark & 8.128 & 8.159 & 8.064 & 7.876 & 7.572 & 7.203 \\
\hline +Lebenserwartung & 8.128 & 8.240 & 8.371 & 8.492 & 8.534 & 8.509 \\
\hline
\end{tabular}

Quelle: eigene Berechnungen basierend auf Statistik Austria Daten. 
Für die volkswirtschaftliche Betrachtung ist nicht nur die Entwicklung der Altenbelastungsquote von Bedeutung, sondern auch die Entwicklung der Bevölkerungsanzahl insgesamt. Beispielsweise sind bei den beiden Varianten Benchmark und +Fruchtbarkeit+Wanderung ähnliche Altenbelastungsquoten $(58 \%$ und 58,4\%) im Jahr 2050 beobachtbar. Die Entwicklung der Gesamtbevölkerungsanzahl divergiert allerdings in den beiden genannten Szenarien sehr stark. In der Benchmark-Variante werden für das Jahr 20506.836 Mio. Personen, für die +Fruchtbarkeit+Wanderung-Variante dagegen 9.734 Mio. Personen prognostiziert. Dies sollte sich in der Prognose des gesamten Steueraufkommens entsprechend widerspiegeln.

\subsubsection{Profile und Steuerarten}

Diese beiden betrachteten Aufkommensarten - Lohnsteuer, auf ganzjährige Einkommen beschränkt, und Umsatzsteuer - stehen im Dreijahresdurchschnitt 1999-2001 für über 60,5\% der gesamten Bruttoabgaben. Die nächstgrößeren Aufkommensarten, die in der Berechnung noch keine Berücksichtigung finden, sind die Körperschaftsteuer (7,9\%), die veranlagte Einkommensteuer (5,8\%), und die Mineralölsteuer (4,9\%).

\section{Lohnsteuer}

Zur Berechnung des Lohnsteueraufkommens wurde die Lohnsteuerstatistik 2001 herangezogen. Mit Alterskohorten verknüpfte Lohnsteuerdaten sind für die Bezieher ganzjähriger Einkommen verfügbar. 2001 betrug das Lohnsteueraufkommen dieser Aufkommensart $€ 15.432$ Mio. ${ }^{13}$ Nicht ganzjährige Einkommensbezieher wurden daher bei den demografischen Berechnungen nicht berücksichtigt. Bezogen auf die Bemessungsgrundlage wurden so bei den Arbeitnehmern 6,8\% und bei den Pensionisten 1,8\% des Aufkommens nicht berücksichtigt.

Die Berechnung der Aufkommensprofile gestaltete sich als verhältnismäßig einfach. In jeder Alterskohorte wurde das durchschnittliche Aufkommen $E$ je Steuerfall ermittelt. Anhand der konstant gehaltenen Fallquote (Verhältnis Steuerfälle $\mathrm{zu}$ gesamter Bevölkerung in der Kohorte) wurde das Lohnsteueraufkommen für die unterschiedlichen demografischen Szenarien berechnet (Tabelle 3). ${ }^{14}$

13 Da die Profile auf dem Jahr 2001 basieren und die demografische Prognose im Jahr 2000 beginnt, kommt es bei der Multiplikation der Lohnsteuerprofile mit den respektiven Alterskohorten $2001 \mathrm{zu}$ leicht unterschiedlichen Startwerten des Steueraufkommens für das Jahr 2001, da sich die Alterskohorten je nach dem demografischen Prognoseszenario schon leicht unterschiedlich von 2000 auf 2001 entwickeln.

14 Ein weiterer Schritt dieser Arbeit könnte die Trennung zwischen Männern und Frauen sein. Dies wäre angesichts der geringeren Beschäftigungsquote bei Frauen interessant. 
Tabelle 3:

Lohnsteuerprofil der Alterskohorten

\begin{tabular}{|c|c|c|c|c|}
\hline \multicolumn{5}{|c|}{ Profil der ganzj. Beschäftigten und Pensionisten } \\
\hline Kohorten & $\begin{array}{l}\text { Arbeitn. } \\
\text { Profil }(€)\end{array}$ & Fallquoten $(\%)$ & Pens. Profil $(€)$ & Fallquoten $(\%)$ \\
\hline bis unter 19 & 360,68 & $1,6 \%$ & 398,43 & $0,1 \%$ \\
\hline 19 bis unter 25 & $1.731,66$ & $38,7 \%$ & 339,86 & $0,3 \%$ \\
\hline 25 bis unter 30 & $3.011,28$ & $49,8 \%$ & 766,40 & $0,1 \%$ \\
\hline 30 bis unter 40 & $4.394,04$ & $53,5 \%$ & 885,29 & $0,2 \%$ \\
\hline 40 bis unter 50 & $5.671,22$ & $57,9 \%$ & $1.371,05$ & $1,2 \%$ \\
\hline 50 bis unter 55 & $6.539,61$ & $50,2 \%$ & $2.342,18$ & $4,8 \%$ \\
\hline 55 bis unter 60 & $8.876,44$ & $29,5 \%$ & $2.822,57$ & $24,5 \%$ \\
\hline 60 bis unter 65 & $14.032,34$ & $6,5 \%$ & $3.423,00$ & $56,5 \%$ \\
\hline 65 bis unter 70 & $17.732,50$ & $0,5 \%$ & $3.245,29$ & $57,5 \%$ \\
\hline 70 und älter & $17.762,39$ & $0,1 \%$ & $3.249,58$ & $59,3 \%$ \\
\hline
\end{tabular}

Quelle: eigene Berechnungen basierend auf Statistik Austria (2002).

\section{Umsatzsteuer}

Die langfristige Entwicklung des Umsatzsteueraufkommens wurde an Hand eines aus der Konsumerhebung 1999/2000 ermittelten Steuerprofils errechnet. Das Umsatzsteuerprofil wurde folgendermaßen ermittelt: Auf die jeweiligen Verbrauchsgruppen der einzelnen Haushaltstypen, weiter untergliedert nach Alterskohorten, wurde die respektive durchschnittliche jährliche Umsatzsteuer aufgerechnet. Aus den Haushalten wurde mit Division durch die Anzahl der Haushaltsmitglieder, Kinder wurden mit 20\% der Haushaltsausgaben gewichtet, das Umsatzsteuerprofil von einzelnen Personen herausgerechnet. ${ }^{15}$ Die Multiplikation der so ermittelten Umsatzsteuerprofile mit allen Alterskohorten lag um etwas weniger als $11 \%$ über dem tatsächlichen Wert. Die Profile wurden in der Folge linear an den tatsächlichen Wert angepasst. Die zur Verfügung gestellten Alterskohorten lagen für die aggregierten Kohorten bis 29 Jahre, 30 bis 44 Jahre, 45 bis 59 Jahre und 60 Jahre und mehr vor. Die respektiven Umsatzsteuerprofilwerte je Person belaufen sich auf $€ 1.741, € 2.427, € 2.358$ und $€ 2.103 .{ }^{16}$

15 Die Gewichtung der Kinder mit 20\% der Haushaltsausgaben geschieht in Anlehnung an Keuschnigg et al. (2000) und Keuschnigg et al. (2002).

16 Bei Betrachtung der Entwicklung der Durchschnittwerte wird deutlich, dass aufgrund der großen Alterskohorten die unterschiedlichen Profile wenig Einfluss auf das Durchschnittsaufkommen haben (Tabelle 6). Eine Gliederung in 5-Jahres-Alterskohorten würde die demografischen Veränderungen wahrscheinlich etwas deutlicher zum Ausdruck bringen. Entsprechende Daten waren nicht verfügbar. 


\section{Konsumbeschränkung}

Im Jahr 2000 betrug der Anteil der Pensionsaufwendungen am BIP 10,5\% bzw. $€ 21,58$ Mrd. (KLP 2002). Der aus der Konsumerhebung herausgerechnete Konsum der über 59-Jährigen beläuft sich auf $€ 22,24 \mathrm{Mrd}$. Beim Hauptszenario wird der Konsum der über 59-Jährigen für 2050 auf $€$ 37,93 Mrd. geschätzt.

Der Konsum der über 59-Jährigen wird aus Arbeitgeber- und Arbeitnehmerbeiträgen aus der Pflichtversicherung mit einem Betrag von anteilig $€ 13,69$ Mrd. finanziert (Abbildung 1). Es wurde der Pflichtbeitrag je Arbeitnehmer (die Summe aus Arbeitnehmer- und Arbeitgeberbeitrag) ermittelt, der dann demografisch fortgeschrieben wurde (Tabelle 4). Daraus resultiert die mögliche Beitragsfinanzierung bei konstant gehaltenen Beitragssätzen über den Beobachtungszeitraum. Der anteilige Bundesbeitrag beträgt $€ 3,7 \mathrm{Mrd}^{17}$ Der anteilige Bundesbeitrag wurde über den Beobachtungszeitraum konstant fortgeschrieben. Im Jahr 2000 ergibt die Differenz aus dem gesamten Konsum und dem Pensionsaufkommen.

\section{Abbildung 1:}

Finanzierung Konsum über 59-jähriger Pensionisten

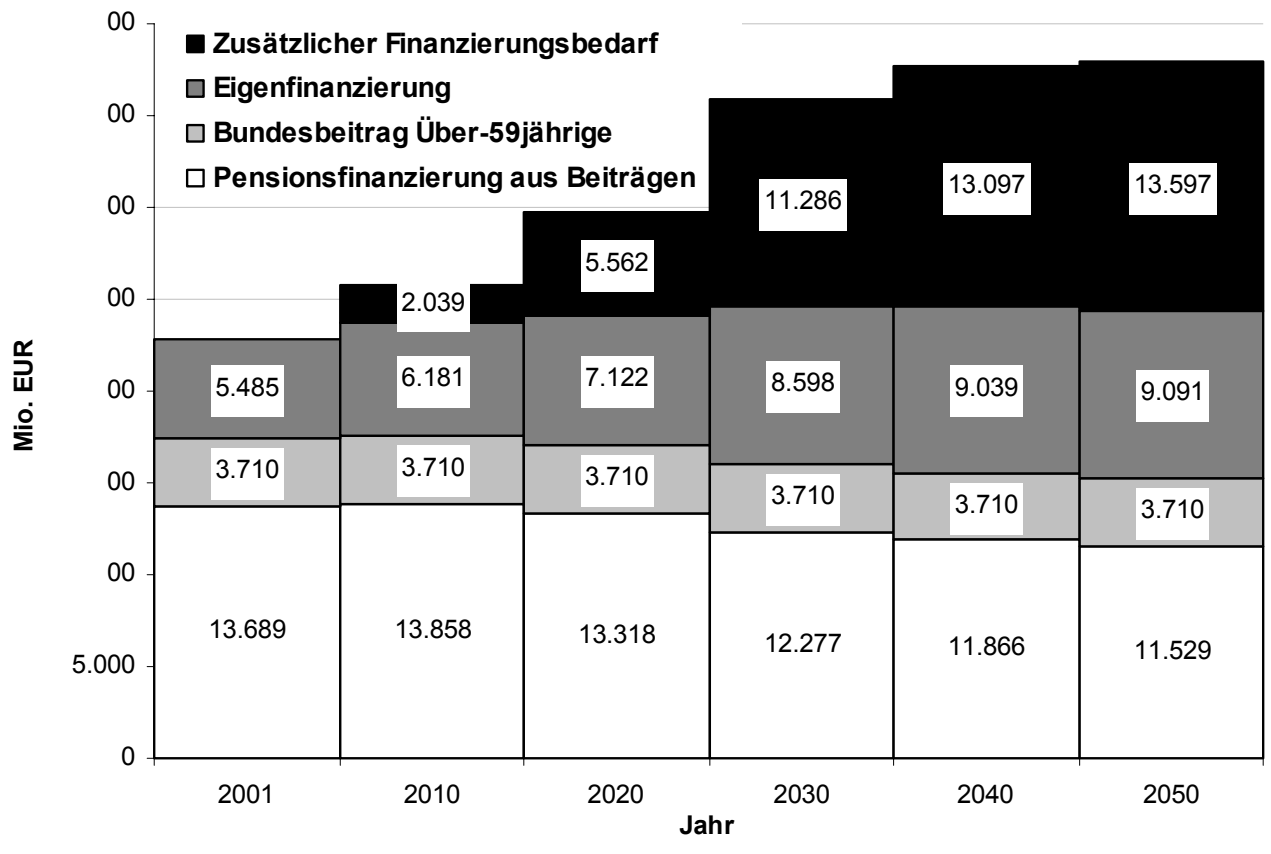

7 In der aktuellen Rechnung wurden lediglich die Beitragszahlungen an die gesetzliche Pensionsversicherung (ASVG, BSVG, GSVG) berücksichtigt. Der Ruhegenuss der Beamten wurde nicht mit einbezogen. In einer noch folgenden Rechnung soll dies berücksichtigt werden. Bei der Rechnung kommt es dadurch zu einer Unterschätzung der Konsumfinanzierung der über 59-Jährigen aus den Pensionsversicherungsbeiträgen. 
aus der 1. Säule jenen Konsum, der aus anderen Quellen (z.B. Auflösen von Ersparnissen, Vermögenstransfers, etc. $)^{18}$ finanziert wird. Daraus wird die Eigenfinanzierung pro Kopf herausgerechnet $(€ 3.184)$, deren Gesamtvolumen ebenfalls entsprechend der Entwicklung der Alterskohorte fortgeschrieben wird.

Tabelle 4:

Profil Pensionsbeiträge

\begin{tabular}{lcc}
\hline Kohorten & Beitragsz. Profil $(€)$ & Beitragsz. Fallquoten \\
\hline bis unter 19 & 1.187 & $5,8 \%$ \\
19 bis unter 25 & 3.466 & $50,8 \%$ \\
25 bis unter 30 & 4.425 & $63,8 \%$ \\
30 bis unter 40 & 4.857 & $70,0 \%$ \\
40 bis unter 50 & 5.374 & $74,7 \%$ \\
50 bis unter 55 & 5.655 & $64,3 \%$ \\
55 bis unter 60 & 6.164 & $37,2 \%$ \\
60 bis unter 65 & 6.245 & $8,2 \%$ \\
\hline
\end{tabular}

Quelle: eigene Berechnungen basierend auf Statistik Austria Daten.

Das geringere Einkommen der Pensionisten bei konstanten Pensionsversicherungsbeiträgen schlägt sich ebenso im Lohnsteueraufkommen nieder. Auf Grund der Progression ist bei fallendem Durchschnittseinkommen mit einem überdurchschnittlichen Rückgang des Steueraufkommens zu rechnen. Mangels der Möglichkeit, diesem Umstand Rechnung tragende Lohnsteuerprofile $\mathrm{zu}$ erstellen, wurde eine vereinfachte Berechnungsvariante des Aufkommensrückgangs gewählt. Die Veränderung des zuvor berechneten ProKopf-Konsums der über 59-Jährigen wurden anteilig (Lohnsteueraufkommen der über 59-Jährigen) proportional auf das Pro-Kopf-Pensionistenaufkommen umgelegt. Angesichts der Progression der Lohnsteuer ist dies sicherlich eine zu optimistische Annahme.

\subsection{Ergebnisse}

\subsubsection{Konstante Profile}

\section{Lohnsteueraufkommen}

Bei den Arbeitnehmern beträgt der Startwert $€ 11,73$ Mrd. im Jahr 2001. Im Hauptszenario steigt dieser Wert leicht auf $€ 12,1$ Mrd. bis 2010 an, um dann kontinuierlich um insgesamt $13,2 \%$ auf $€ 10,18$ Mrd. zu sinken. Bei der

18 Auch Einkünfte aus der Eigenvorsorge - mit steigender Tendenz in den kommenden Jahren können zur Eigenfinanzierung beitragen (siehe Tabelle AN1 in Annex). 
+Fruchtbarkeit+Wanderung-Variante ist bis 2050 ein leichtes Wachstum von 2\% auf knapp $€ 11,96$ Mrd. möglich. In der -Fruchtbarkeit-Wanderung-Variante sinkt dieser Wert um etwas weniger als $28 \%$ auf $€ 8,48 \mathrm{Mrd}$.

Dieser wahrscheinliche Rückgang wird von der Entwicklung des Lohnsteueraufkommens aus den Pensionen mehr als ausgeglichen. Im Hauptszenario steigt die Lohnsteuer aus den Pensionen von $2001 € 3,7$ Mrd. auf $€ 5,92$ Mrd. Im schlechtesten Fall (Benchmark-Variante) immer noch auf $€ 4,87$ Mrd.

Für das gesamte Lohnsteueraufkommen bedeutet dies, dass im Hauptszenario von 2001 bis 2050 eine Steigerung um 4,36\% auf $€ 16,1 \mathrm{Mrd}$. erwartet werden kann. Getragen wird diese Steigerung ausschließlich durch einen äußerst starken Anstieg der Lohnsteuer aus den Pensionen. Ein Sinken des gesamten Aufkommens kann nur im Benchmark-Szenario $(-€ 0,8$ Mrd.) und im -Fruchtbarkeit-Wanderung-Szenario (-€ 1,4 Mrd.) beobachtet werden.

\section{Umsatzsteueraufkommen}

Im Hauptszenario steigt das Umsatzsteueraufkommen von 17,06 Mrd. im Jahr 2000 leicht auf $€$ 17,3 Mrd. im Jahr 2050 an. Ein Absinken des Aufkommens kann nur in der -Fruchtbarkeit-Wanderung-Variante (-€ 3 Mrd.) und in der BenchmarkVariante $(-€ 1,8$ Mrd.) beobachtet werden. Ähnlich dem Lohnsteueraufkommen wird die Steigerung des Umsatzsteueraufkommens wesentlich von der Hebung des Anteils der über 59-Jährigen getragen. In der obersten Alterskohorte steigt das Aufkommen in der Hauptvariante von $€ 3,52$ Mrd. auf 6 Mrd. an. Die Entwicklung des durchschnittlichen Pro-Kopf-Aufkommens zeigt, dass das Umsatzsteueraufkommen wesentlich stärker von der Änderung der Gesamtbevölkerung abhängt als von der Verschiebung der Alterskohorten, über den Beobachtungszeitraum konstant gehaltene Umsatzsteuerprofile vorausgesetzt.

\section{Gesamtaufkommen}

Wie zuvor erwähnt beträgt der Anteil des auf demografische Veränderungen untersuchten Steueraufkommens gemessen am Bruttoabgabenaufkommen knapp über $60 \%$. Im Hauptszenario steigert sich die Summe aus Lohn- und Umsatzsteueraufkommen von $€ 32,79$ Mrd im Jahr 2001 auf $€ 33,4$ Mrd. Gemessen am gesamten Bruttoabgabenaufkommen von $€ 56,21$ Mrd. macht dies eine Steigerung von $1,1 \%$ aus. Im ungünstigsten -Fruchtbarkeit-WanderungSzenario zeichnet die Veränderung des Umsatz- und Lohnsteueraufkommens für einen Rückgang von 7,5\% der Bruttoabgaben verantwortlich.

Die Summe des Steueraufkommens aus Pensionen und des UmsatzsteuerAufkommens der Kohorte der über 59-Jährigen steigt im Hauptszenario von 7,29 auf $€ 11,93$ Mrd. Je nach demografischer Prognosevariante steigt der Anteil der obersten Alterskohorte von 22,2\% im Jahr 2001 auf zwischen 32,7\% (Benchmark) und 39,4\% (-Fruchtbarkeit-Wanderung). In der Hauptvariante beträgt dieser Anteil $35,7 \%$, bzw. eine Anteilssteigerung von mehr als $60 \%$. 
Allein schon aus Betrachtung der beiden hochgerechneten Steuerarten wird deutlich, dass eine Diskussion des Aufkommens nicht abgekoppelt von Ausgabenbetrachtungen erfolgen kann. Kommt es beispielsweise zu Erhöhungen der Gesundheitsausgaben, wie oftmals prognostiziert, und damit der Sozialversicherungsbeiträge, wird dies spürbare Rückgänge im restlichen besteuerbaren Konsum und auch Rückgänge bei der Bemessungsgrundlage der Lohnsteuer mit sich bringen. Eine ebensolche Logik gilt für die Pensionsversicherungsbeiträge. Erhöhte Pensionsversicherungsbeiträge werden bis zu einem gewissen Grad zu Konsumverzicht (zu einem gewissen Teil auch zum Entsparen) führen. Tendenzen zum Rückgang der einen oder anderen Bemessungsgrundlage scheinen unausweichlich.

\subsubsection{Konsumbeschränkung}

Aufkommensrückgänge sind nun im Hauptszenario sowie weiteren drei Prognosevarianten sowohl beim Umsatzsteueraufkommen der über 59-Jährigen und des Lohnsteueraufkommens der Pensionisten die Folge.

\section{Umsatzsteueraufkommen}

Bei Fortschreibung des Konsumprofils der über 59-Jährigen ergibt sich bis 2050 unter obigen Annahmen eine Finanzierungslücke von $€ 13,6 \mathrm{Mrd}$. (35,9\%) für einen geschätzten Konsum von $€ 37,93$ Mrd. Wenn die Finanzierung für diesen zusätzlichen Konsum nicht aufgebracht werden kann, ist mit einem proportionalen Einbruch des Umsatzsteueraufkommens der über 59-Jährigen im Hauptszenario zu rechnen. Das gesamte geschätzte Umsatzsteueraufkommen für 2050 würde sich um $€ 2,16$ Mrd. auf $€ 15,14$ Mrd reduzieren. Die Entwicklung des Umsatzsteueraufkommens würde somit eine deutliche negative Entwicklung von 2000 an verzeichnen $(-11,2 \%)$. Der größte Einbruch resultiert bei der -Fruchtbarkeit-Wanderung-Variante. Im Zeitraum 2000 bis 2050 würde das Aufkommen um $€ 28,8 \%$ auf 12,14 Mrd. einbrechen. In der + Fruchtbarkeit + Wanderung-Variante würde das Aufkommen im gleichen Zeitraum um 7,81\% auf $€ 18,38 \mathrm{Mrd}$. anwachsen.

\section{Lohnsteueraufkommen}

In der Hauptvariante nimmt das Lohnsteueraufkommen der Pensionisten bis 2050 um 6,89\% auf $€ 3,96$ Mrd. zu (Tabelle 5). Die Summe aus Lohnsteuer der Arbeitnehmer und der Pensionisten nimmt nun aber 8,36\% auf $€ 14,1$ Mrd bis $2050 \mathrm{ab}$ (gegenüber einem Startwert von $€ 15,43 \mathrm{Mrd}$. im Jahr 2001). Der stärkste Einbruch des Lohnsteueraufkommen wird wieder in der -Fruchtbarkeit-Wanderung-Variante verzeichnet. Das Lohnsteueraufkommen sinkt hier um 22,2\% auf $€ 12$ Mrd. ab. Die einzige Variante, in der es zu einer Steigerung des gesamten Lohnsteueraufkommens kommt, ist die 
+ Fruchtbarkeit + Wanderung-Annahme. Das Aufkommen steigt hier um 6\% auf $€ 16,36$ Mrd. im Jahr 2050.

\section{Gesamtaufkommen}

Unter Berücksichtigung der Konsumbeschränkung sinkt das Aufkommen der Lohn- und Umsatzsteuer in der Hauptvariante von einem Anfangswert von $€ 32,79$ Mrd. im Jahr 2001 demografisch bedingt auf $€ 29,28$ Mrd. Dies entspricht einer Reduktion des Startwertes um 10,7\%, oder einer Verringerung des gesamten Bruttoaufkommens um 6,2\%. Der stärkste Einbruch ist in der -Fruchtbarkeit-Wanderung-Variante beobachtbar. In dieser Variante sorgt die demografische Entwicklung dafür, dass das Bruttoabgabenaufkommen durch Abnahmen im Lohn- und Umsatzsteueraufkommen um 15,4\% einbricht. In der + Fruchtbarkeit + Wanderung-Variante macht die lohn- und umsatzsteuerbedingte Steigerung an den Bruttoabgaben 2001 3,5\% aus.

Tabelle 5:

Lohnsteuerentwicklung ganzjähriger Einkommen gegliedert in Arbeitnehmer und Pensionisten

\begin{tabular}{lcccccc}
\hline $\begin{array}{l}\text { Lohnst. ganzj. Beschäftiger \& } \\
\text { Pensionisten in Mio. } €\end{array}$ & $\mathbf{2 0 0 1}$ & $\mathbf{2 0 1 0}$ & $\mathbf{2 0 2 0}$ & $\mathbf{2 0 3 0}$ & $\mathbf{2 0 4 0}$ & $\mathbf{2 0 5 0}$ \\
\hline Pensionisten & & & & & & \\
\hline Haupt & 3.703 & 4.156 & 4.839 & 5.644 & 5.892 & 5.920 \\
-Fruchtbarkeit -Wanderung & 3.703 & 4.149 & 4.800 & 5.541 & 5.685 & 5.566 \\
+Fruchtbarkeit +Wanderung & 3.703 & 4.164 & 4.878 & 5.746 & 6.098 & 6.275 \\
Benchmark & 3.702 & 4.093 & 4.603 & 5.134 & 5.084 & 4.879 \\
+Lebenserwartung & 3.703 & 4.178 & 4.922 & 5.832 & 6.232 & 6.454 \\
\hline Abweichung haupt von Wert 2001 (\%) & $12,26 \%$ & $30,70 \%$ & $52,42 \%$ & $59,12 \%$ & $59,90 \%$ \\
\hline Summe Arbeitnehmer \& Pensionisten & & & & & \\
\hline Haupt & 15.432 & 16.253 & 16.687 & 16.566 & 16.372 & 16.105 \\
-Fruchtbarkeit -Wanderung & 15.432 & 16.165 & 16.314 & 15.735 & 14.954 & 14.041 \\
+Fruchtbarkeit +Wanderung & 15.433 & 16.341 & 17.060 & 17.422 & 17.831 & 18.238 \\
Benchmark & 15.431 & 16.171 & 16.392 & 15.943 & 15.321 & 14.609 \\
+Lebenserwartung & 15.433 & 16.280 & 16.787 & 16.784 & 16.756 & 16.697 \\
\hline Konsumbeschränkung Summe Arbeitnehmer und Pensionisten & & \\
\hline Haupt & 15.432 & 15.959 & 15.885 & 14.939 & 14.483 & 14.142 \\
-Fruchtbarkeit -Wanderung & 15.432 & 15.861 & 15.475 & 14.038 & 12.974 & 12.007 \\
+Fruchtbarkeit +Wanderung & 15.433 & 16.058 & 16.296 & 15.873 & 16.040 & 16.362 \\
Benchmark & 15.431 & 15.922 & 15.757 & 14.676 & 13.987 & 13.341 \\
+Lebenserwartung & 15.433 & 15.971 & 15.926 & 15.020 & 14.616 & 14.340 \\
\hline Abweichung haupt von Wert 2001 (\%) & $3,41 \%$ & $2,93 \%$ & $-3,20 \%$ & $-6,15 \%$ & $-8,36 \%$ \\
\hline
\end{tabular}




\section{Wirtschaftspolitische Implikationen}

Die zuvor analysierte demografisch bedingte Veränderung des Steueraufkommens berücksichtigt die zwei größten österreichischen Aufkommensarten, die einen Anteil von etwas mehr als $60 \%$ an den gesamten Bruttoabgaben haben. Bei den demografisch ungünstigen Varianten ist mit spürbaren Rückgängen gemessen an den Bruttoabgaben $\mathrm{zu}$ rechnen. Mit demografisch bedingten Aufkommensreduktionen ist ab der zweiten Hälfte der zweiten Dekade zu rechnen. Die Entwicklung wesentlicher Aufkommensarten wie der veranlagten Einkommensteuer, die sich demografisch bedingt ähnlich wie die Lohnsteuer entwickeln könnte, der Körperschaftsteuer und der Mineralölsteuer wurden bei der einfachen Modellrechnung nicht berücksichtigt. Nichtsdestotrotz kann man mit großer Wahrscheinlichkeit davon ausgehen, dass der Bedarf besteht, alternative bzw. ergänzende Einnahmequellen auszumachen, insbesondere durch die zu erwartende steigende Mobilität auch beim Faktor Arbeit.

Der prognostizierte Rückgang beim Steueraufkommen resultiert vor allem aus dem sinkenden Anteil der Erwerbstätigen. Die Erwerbsquote entsprechend anzuheben, um dieser Entwicklung entgegen zu wirken, muss daher das Ziel sein. Eine Anhebung der Lohnsteuer würde Impulse setzen, die der Anhebung der Erwerbsquote entweder entgegen laufen und/oder zu einer Abdrängung in die Schattenwirtschaft mit entsprechenden Konsequenz für das Aufkommen führen können. Die konjunkturdämpfende Wirkung einer solchen Maßnahme täte ein Übriges, um das ohnehin schon als schwächer prognostizierte Wachstum weiter zu drücken. Auch muss in Anbetracht steigender Mobilität von Arbeitskräften bei steigenden Steuern von entsprechenden Ausweichreaktionen à la ,voting with their feet" ausgegangen werden. ${ }^{19}$

Weiters ist festzuhalten, dass Österreich gemessen am BIP das sechsthöchste USt-Aufkommen innerhalb der EU-25 aufweist. In der Periode 1995-2002 betrug der EU-Jahresdurchschnitt 7,0\%, während sich die entsprechende Quote für Österreich auf $8,2 \%$ beläuft (EC 2004, 242). Durch die verhältnismäßig hohe Abhängigkeit vom USt-Aufkommen ist der durch die Konsumbeschränkungen prognostizierte Einbruch für Österreich vergleichsweise bedeutsam. Auch ist vor diesem Hintergrund eine Anhebung des USt-Satzes zur Stabilisierung des Aufkommens wenig praktikabel, einerseits wegen des bereits relativ hohen UStSatzes und andererseits durch die konsumdämpfende Wirkung einer Anhebung des USt-Satzes. Letztlich sollten daraus die Bestrebungen resultieren, die Ausgabenströme der Pensionisten zu stabilisieren, wenn nicht gar zu stärkerem Konsum anzuregen.

19 In diesem Zusammenhang sei darauf hingewiesen, dass aus der Debatte zum fiskalen Föderalismus ebenfalls Einsichten zur Schaffung alternativer Steueraufkommen gewonnen werden können. Vgl. dazu Heft 4/2004 der Wirtschaftspolitischen Blätter mit dem Schwerpunkt „Fiskaler Föderalismus“. 
Von der Berücksichtigung des KöSt-Aufkommens in der vorliegenden Berechnung wurde unter anderem deshalb Abstand genommen, da der Zusammenhang zwischen der demografischen Entwicklung und dem KöStAufkommen kein unmittelbarer ist. Bei Aufaddieren des KöSt-Aufkommens und der Erwerbstätigen der ÖNACE-Positionen A, B, C, F, K, L, M, N, O stellt das KöSt-Aufkommen dieser Sektoren 23,1\% des gesamten KöSt-Aufkommens und die Zahl der Erwerbstätigen aus diesen Sektoren 53,4\% aller Erwerbstätigen dar. Der Zusammenhang zwischen der Anzahl der Erwerbstätigen und dem KöStAufkommen dürfte gering bzw. nicht gegeben sein. Für das KöSt-Aufkommen ist insbesondere der internationale Steuerwettbewerb von Bedeutung. Der KöSt-Satz wurde in Österreich während der letzten Jahre zwei Mal gesenkt, wobei langfristig nicht absehbar ist, ob sich der „race to the bottom“ auch während der nächsten Jahrzehnte noch fortsetzen wird. Der aktuelle KöSt-Satz beträgt in Österreich 25\%, jener von Irland 12,5\%. Potenziell sind durch Steuerwettbewerb Satzsenkungen möglich (gemessen am irischen KöSt-Satz 50\%), die sehr wahrscheinlich weit über dem Einfluss der demografischen Entwicklung der Erwerbsbevölkerung liegen. In der Literatur gilt es als allgemein gesichert, dass der Zusammenhang zwischen der Höhe der KöSt-Besteuerung und den ausländischen Direktinvestitionen negativ ist. ${ }^{20}$ Es wird zudem in einigen Arbeiten davon ausgegangen, dass der Einfluss des KöSt-Satzes auf die Investitionsentscheidung verhältnismäßig schwach ist. Es ist daher schwer abzuschätzen, ob langfristig weiterhin das Argument des Steuerwettbewerbs dominiert oder ob die Einschätzung des schwachen Zusammenhangs zwischen der KöSt und Standortentscheidungen von der Politik ebenfalls geteilt wird.

In der Modellrechnung wurde von einem Nullwachstum ausgegangen. Wenn von dieser Annahme abgewichen wird und beispielsweise alternativ ein Produktivitätswachstum von $0,5 \%$ angenommen wird- dies entspricht den pessimistischsten Wachstumsannahmen bedingt durch die demografischen Veränderungen - würde dieses Wachstum ausreichen, um den prognostizierten Aufkommensrückgang von $0,35 \%$ jährlich bei einer Aufkommenselastitzität von 1 zu kompensieren. Ein Teil des Wachstums muss dazu verwendet werden, den Lebensstandard (in Form öffentlicher Leistungen) lediglich zu halten, statt dass das Wachstum wie unter günstigeren Altenbelastungsquoten zur Gänze eine Erhöhung des Lebensstandards bewirken würde.

Abschließend ist noch $\mathrm{zu}$ berücksichtigen, wie die aus der Generationenrechnung gewonnenen Aussagen in der Politik Berücksichtigung finden. In der Öffentlichkeit wird in der Regel der expliziten Verschuldung mehr Aufmerksamkeit geschenkt als der impliziten Verschuldung, wie sie die Generationenrechnung aufzeigt (Weil 2001). Als Beispiel für Österreich kann angeführt werden, dass die Implikationen aus dem demografischen Wandel für das

\footnotetext{
${ }^{20}$ Vergleiche dazu Breuss und Schratzenstaller (2004), de Mooij und Ederveen (2001), Loretz et al. (2004).
} 
umlagenfinanzierte Pensionssystem bereits über viele Jahre bekannt waren, ${ }^{21}$ wirksame Pensionsreformmaßnahmen sehr spät und unter großem finanziellen Druck auf die (explizite) Verschuldung unternommen wurden. Die Frage ist erlaubt, wie die „frühe“ Erkenntnis eines zwar nicht dramatischen aber dennoch spürbaren Rückgangs des langfristigen Steueraufkommens von der Politik aufgegriffen wird.

\section{Literaturverzeichnis}

Bach, S., C. Bork, P. Krimmer, B. Raffelhüschen und E. Schulz. 2002. Demographischer Wandel und Steueraufkommen. Endbericht. Forschungsauftrag 16/01. Berlin: Deutsches Institut der Wirtschaft. Internet: $<<$ http://www.diw.de/deutsch/produkte/publikationen/materialien/docs/papers/diw_rn0 2-11-20.pdf $>>$

Baumgartner, J., H. Hofer, S. Kaniovski, U. Schuh und T. Url. 2004. A Long-run Macroeconomic Model of the Austrian Economy (A-LMM) - Model Documentation and Simulations, Wifo Working Papers 224/2004, Wien: Österreichisches Institut für Wirtschaftsforschung.

Breuss, F. und M. Schratzenstaller. 2004. Unternehmenssteuerwettbewerb und internationale Direktinvestitionen, Wifo Monatsberichte 8/2004, pp. 645-653.

Chaloupek, G., J. Lamel und J. Richter. 1988. Bevölkerungsrückgang und Wirtschaft, Heidelberg: Physika.

Dang, T. T., P. Antolin und H. Oxley. 2001. Fiscal Implications of Ageing: Projections of Age-related Spending. Economic Department Working Papers No. 305, Paris: OECD.

De Mooij, R. A. und S. Ederveen. 2001. Taxation and Foreign Direct Investment: A Synthesis of Empirical Research, CESifo Working Paper No. 558, München: Center for Economic Studies \& Ifo Institute for Economic Research.

Denton, F. T. und B. G. Spencer. 1999. Population Ageing and Its Economic Costs: A Survey of the Issues and Evidence. SEDAP Research Paper 1, Ontario: McMaster University.

EC. 2004. Structures of the taxation systems in the European Union - Data 1995 - 2002, Luxemburg: European Communities.

Group of Ten. 1998. The macroeconomic and financial implications of ageing populations, Group of Ten April 1998, Basel: Bank for International Settlement.

Gruber, J. und D. Wise 2001. An International Perspective on Policies for an Ageing Society. NBER Working Paper w8103. Cambridge: National Bureau of Economic Research.

Hanika, A. 2000, Bevölkerungsvorausschätzung 2000-2050 für Österreich und die Bundesländer, Statistische Nachrichten 12: 977-989.

Holzmann, R. 2000. Alterssicherung und Finanzmärkte - können Investitionen in den Emerging Markets helfen, das Überalterungsproblem in den westlichen Ländern zu lösen? Wirtschaftspolitische Blätter 4: 407-417.

21 Für eine frühe Analyse der Auswirkungen des demografischen Wandels auf die österreichische Wirtschaft siehe Chaloupek et al. (1988). 
Keuschnigg, C., M. Keuschnigg, R. Koman, E. Lüth und B. Raffelhüschen. 2002. Intergenerative Inzidenz der österreichischen Finanzpolitik, in: E. Theurl, H. Winner, und R. Sausgruber (Eds.) Kompendium der österreichischen Finanzpolitik. Wien: Springer Verlag, S. 263-295.

Keuschnigg, C., M. Keuschnigg, R. Koman, E. Lüth und B. Raffelhüschen. 2000. Public Debt and Generational Balance in Austria, Economic Series 80, Wien: Institut für Höhere Studien.

Keller, C. 2000. Die Zukunft der gesetzlichen Rentensysteme in der europäischen Union, Untersuchungen zur Wirtschaftspolitik 118. Köln: Institut für Wirtschaftspolitik an der Universität zu Köln.

KLP. 2002. Gutachten der Kommission zur langfristigen Pensionssicherung über die längerfristige Entwicklung der gesetzlichen Pensionsversicherung in den Jahren 2000 bis 2050. Wien: Kommission zur langfristigen Pensionssicherung.

Külp, B. 1993. Das Mackenroth-Theorem. Wirtschaftswissenschaftliches Studium 2: 82-84.

Lehner, G. 2002. Längerfristige Entwicklung der Steuern und Abgaben in Österreich, Monografien 11. Wien: Österreichisches Institut für Wirtschaftsforschung.

Lehner, G. 2003. Längerfristige Entwicklung der Steuern und Abgaben in Österreich, Wirtschaftspolitische Blätter 2: 236-245.

Leibrecht, M. 2002. Steueraufkommensprognose in Österreich - Ablauf, Methoden und Präzision der Prognose. Wirtschaftsuniversität Wien, Dissertation.

Loretz, S., M. Pfaffermayr, und H. Winner. 2004. Unternehmensbesteuerung, internationale Direktinvestitionen und die Steuerreform 2005, Wirtschaftspolitische Blätter 4: 495-505.

Mackenroth, G. 1952. Die Reform der Sozialpolitik durch einen deutschen Sozialplan. Schriften des Vereins für Socialpolitik 24: 37-76.

Raffelhüschen, B. 1999. Generational Accounting: Method, Data and Limitations. In: European Commission (Ed.) Generational Accounting in Europe. European Economy 6. Brüssel.

Statistik Austria. 2002. Statistik der Lohnsteuer 2001. Statistik Austria, Wien.

Venti, S. F. und D. A. Wise. 2001. Aging and Housing Equity: Another Look. NBER Working Paper w8608. Cambridge: National Bureau of Economic Research.

Visco, I. 2001. The Fiscal Implications of Ageing Populations in OECD Countries, In: Pensions Symposium: Markets, Policies and People, Worcester College 7. Juni 2001. Worcester: Oxford Centre on Population Ageing.

Wagner, G., V. Meinhart, J. Leinert und E. Kirner. 1998. Kapitaldeckung: Kein Wundermittel für die Vorsorge. DIW Wochenbericht 46. Berlin.

Weil, D. 2001. Comment on: Raffelhüschen, Bernd.Aging, Fiscal Policy, and Social Insurance: A European Perspective: In: A. Auerbach und R. Lee. (Eds.) Demographic Change and Fiscal Policy. Cambridge: Cambridge University Press, pp. 243-247.

Werding, M. 1998. The Pay-as-you-go Mechanism as Human Capital Funding: The Mackenroth Hypothesis Revisited, Passauer Diskussionspapiere V-7-98. Passau: Universität Passau. 


\section{ANNEX}

Tabelle AN1:

Entwicklung des Ust-Aufkommens Absolutwerte, Durchschnittswerte und Anteil über 59Jähriger

\begin{tabular}{lcccccc}
\hline $\begin{array}{l}\text { Entwicklung des Ust- } \\
\text { Aufkommens in Mio } €\end{array}$ & $\mathbf{2 0 0 0}$ & $\mathbf{2 0 1 0}$ & $\mathbf{2 0 2 0}$ & $\mathbf{2 0 3 0}$ & $\mathbf{2 0 4 0}$ & $\mathbf{2 0 5 0}$ \\
\hline Haupt & 17.056 & 17.369 & 17.589 & 17.679 & 17.567 & 17.295 \\
-Fruchtbarkeit -Wanderung & 17.056 & 17.160 & 16.825 & 16.254 & 15.472 & 14.507 \\
+Fruchtbarkeit +Wanderung & 17.056 & 17.627 & 18.386 & 19.176 & 19.827 & 20.337 \\
Benchmark & 17.056 & 17.235 & 17.085 & 16.685 & 16.042 & 15.262 \\
+Lebenserwartung & 17.056 & 17.396 & 17.691 & 17.904 & 17.916 & 17.967 \\
\hline Abweichung haupt von Wert 2001 (\%) & $1,83 \%$ & $3,12 \%$ & $3,66 \%$ & $2,99 \%$ & $1,40 \%$ \\
\hline Konsumbeschränkung Ust-Aufkommen & & & & & \\
\hline Haupt & 17.056 & 17.046 & 16.824 & 15.893 & 15.493 & 15.142 \\
-Fruchtbarkeit -Wanderung & 17.056 & 16.825 & 16.020 & 14.359 & 13.228 & 12.144 \\
+Fruchtbarkeit +Wanderung & 17.056 & 17.316 & 17.663 & 17.501 & 17.922 & 18.388 \\
Benchmark & 17.056 & 16.957 & 16.446 & 15.144 & 14.328 & 13.550 \\
+Lebenserwartung & 17.056 & 17.058 & 16.885 & 16.033 & 15.705 & 15.609 \\
Abweichung haupt von Wert 2001 (\%) & $-0,06 \%$ & $-1,36 \%$ & $-6,82 \%$ & $-9,16 \%$ & $-11,22 \%$ \\
\hline
\end{tabular}

Quelle: eigene Berechnungen basierend auf Statistik Austria Daten. 